Sains Malaysiana 49(3)(2020): 703-712

http://dx.doi.org/10.17576/jsm-2020-4903-25

\title{
Modeling the Volatility of Cryptocurrencies: An Empirical Application of Stochastic Volatility Models
}

(Pemodelan Kemeruapan Mata Wang Kripto: Pengaplikasian Empirik Model Kemeruapan Stokastik)

\author{
MAMOONA ZAHID \& FARHAT IQBAL*
}

\begin{abstract}
This paper compares a number of stochastic volatility (SV) models for modeling and predicting the volatility of the four most capitalized cryptocurrencies (Bitcoin, Ethereum, Ripple, and Litecoin). The standard SV model, models with heavy-tails and moving average innovations, models with jumps, leverage effects and volatility in mean were considered. The Bayes factor for model fit was largely in favor of the heavy-tailed SV model. The forecasting performance of this model was also found superior than the other competing models. Overall, the findings of this study suggest using the heavy-tailed stochastic volatility model for modeling and forecasting the volatility of cryptocurrencies.
\end{abstract}

Keywords: Bayesian model comparison; cryptocurrency; jumps; leverage; stochastic volatility

ABSTRAK

Kertas ini membandingkan beberapa model kemeruapan stokastik (SV) untukpemodelan dan penganggaran kemeruapan empat modal mata wang kripto (Bitcoin, Ethereum, Ripple dan Litecoin). Model standard SV, model dengan hujung berat dan inovasi purata pergerakan, model dengan lompatan, kesan pengaruh dan kemeruapan dalam min diambil kira. Faktor Bayes untuk model penyuaian selalunya menyebelahi model SV hujung berat. Prestasi peramalan model ini didapati superior daripada model lain yang dibandingkan. Secara keseluruhan, keputusan kajian ini mencadangkan penggunaan model kemeruapan stokastik hujung berat untuk pemodelan penganggaran kemeruapan mata wang kripto.

Kata kunci: Kemeruapan stokastik; lompatan; mata wang kripto; perbandingan model Bayesi; pengaruh

\section{INTRODUCTION}

Cryptocurrencies are found highly volatile and show extreme tail events as compared to traditional currencies. Accurate forecasts of volatility and hence Value-at-Risk is of vital importance for better decision of investors, practitioners, and policymakers. It is also important to create a model capable of understanding the unique characteristics and dynamics of cryptocurrencies by using traditional and novel techniques (Peng et al. 2018). Bitcoin is one of the most important digital currencies with a market capitalization exceeding $\$ 140$ billion and representing more than $60 \%$ of the total cryptocurrencies capitalization in June 2018 (https://coinmarketcap.com). There are more than 2000 cryptocurrencies. Ethereum, Ripple, and Litecoin are other important digital currencies among top ten cryptocurrencies. These cryptocurrencies are characterized by high volatility and some large shocks and jumps.

Bitcoin, introduced by Nakamoto (2009), is the most popular and prominent decentralized cryptocurrecy. Ethereum is another popular decentralized cryptocurrency. The joining of 86 new members to Enterprise Ethereum in 2017 made it gained significant momentum through the first half of 2017. Litecoin, a leading rival of Bicoin, was created in 2011 by Charles Lee. One of the main features of Litecoin that make it particularly attractive in time- critical situations is its significantly faster confirmation time for transactions. The transactions in Litecoin can be done in minutes rather than hours and hence become a popular choice for payment. Ripple, developed in 2012, is not a fully decentralized cryptocurrency but enables bank to send real-time international payment across networks. One feature of Ripple that gives it an endge over Bitcoin is its greater control over the system and it is not subject to the price volatility of the underlying currencies.

The Generalized Autoregressive Conditional Heteroscedastic (GARCH) model of Bollerslev (1986) and its variants are famous volatility models for modeling the exchange rate of traditional as well as cryptocurrencies. Most of the literature on cryptocurrencies focus on modeling the volatility through GARCH-type models. A major reason for the popularity of GARCH-type models for describing the dynamics of cryptocurrencies volatility is their deterministic dependence of the conditional variance on past observations which enables simple estimation of parameters.

Research on cryptocurrencies other than Bitcoin is limited (Gkillas \& Katsiampa 2018). Several GARCH models with Gaussian errors were employed by Katsiampa (2017) to estimate the volatility of Bitcoin and the AR(1)CGARCH(1,1) model was found the best. Using GARCH models with different error distributions, Chu et al. (2017) 
concluded that the $\operatorname{IGARCH}(1,1)$ model estimates the Bitcoin volatility better than the competing models. Liu et al. (2017) reported the superior performance of the GARCH model with Student- errors than GARCH models with Gaussian and reciprocal inverse Gaussian errors. Urquhart (2017) concluded that the forecasting capability of heterogeneous autoregressive models is superior to GARCH models and found no evidence of the leverage effect in the Bitcoin market. Univariate and multivariate GARCH models and vector autoregressive specifications were also used in literature to study the dynamic properties of Bitcoin (Stavroyiannis \& Babalos 2017). The relationship between Bitcoin price returns and volatility were investigated through asymmetric GARCH models by Bouri et al. (2017).

Cheong et al. (2012) aimed to acquire an appropriate asymmetric time-varying volatility model for the forecast evaluations based on intraday and interday data and found that the threshold GARCH model showed the superior in-sample whereas the power ARCH model showed the superior out-of-sample forecasts. Naimy and Hayek (2018) evaluated the forecasting performance of the standard GARCH and exponential GARCH (EGARCH) models with Gaussian, generalized error and Student- distributions and concluded the superior predictive ability of the EGARCH model. Catania et al. (2018) compared the Gaussian GARCH model with the generalized autoregressive score (GAS) models of Creal et al. (2013) and Harvey (2013). The GAS model was found to outperform the standard GARCH model. Peng et al. (2018) compared both the symmetric $(\mathrm{GARCH})$ and asymmetric (EGARCH and GJR-GARCH) models against the Support Vector Regression GARCH (SVR-GARCH) model of Bezerra and Albuquerque (2017). The symmetric and asymmetric Gaussian and Student- errors were assumed and the volatility forecasts were compared. The results showed that the SVR-GARCH model yields more accurate forecasts. Few studies take into account the presence of outliers to estimate the volatility of Bitcoin (Catania \& Grassi 2017; Catania et al. 2018; Charles \& Darne 2018). Rahim et al. (2018) used variance targeting estimator (VTE) for the GJR-GARCH model and found that this estimator can be used as an alternative estimator for GARCH-type models.

Few studies have employed varieties of GARCH-type models for a number of cryptocurrencies with the aim to select the best volatility model or a superior set of models (Baur \& Dimpfl 2018; Caporale \& Zekokh 2019; Charfeddine \& Maouchi 2018; Peng et al. 2018). Charle and Darne-Lemna (2019) considered comparing simple GARCH-type models without taking into account the asymmetric and long-memory effects related properties associated with the Bitcoin and concluded that these simple models seem not to be appropriate for modeling the Bitcoin returns. Borri (2019) modeled the conditional tail-risk in four major cryptocurrencies and the results showed that these cryptocurrencies are highly exposed to tail-risk within the crypto market contexts. Fakhfekh and Jeribi
(2020) applied various GARCH-type models with different error distributions to sixteen of the most popular cryptocurrencies and found that TGARCH model with double exponential distribution provided the best fit.

Alternatively, stochastic volatility (SV) models introduced by Taylor (1986), where volatility is allowed to evolve according to some latent stochastic process, can be used for modeling the volatility of financial time series. These models provide greater flexibility in describing stylized facts of financial time series (see Ghysels et al. 1996; Shephard 1996) for review of SV models and their applications). Various extensions of the basic SV model of Taylor (1986) have been proposed. These include the model with leverage effect (Harvey \& Shephard 1996), model with heavy-tailed distributions (Liesenfeld \& Jung 2000), volatility in mean model (Koopman \& Hol Uspensky 2002), model with jumps (Eraker et al. 2003), the model with moving average innovations (Chan 2013), among others. However, the estimation of these models is more complicated than GARCH-type models due to the stochastic evolution of volatility.

To the best of our knowledge, no previous studies have applied a number of SV models for the estimation and prediction of conditional volatility of cryptocurrencies. We aim to fill this gap by investigating the volatility dynamics of four popular cryptocurrencies using a number of stochastic volatility models. The basic SV model, SV with Student- errors, SV with jumps, SV with leverage, SV with moving average innovations and SV with volatility in mean models were included in this study. In order to assess the evidence in favor of more flexible SV models against the standard SV model given the data of cryptocurrencies, a formal Bayesian model comparison exercise was carried out. One step ahead forecasts of conditional volatility generated from each model were also evaluated through the Diebold-Mariano test.

Findings of this study can be briefly summarized as follows: The stochastic volatility model with heavy-tailed is found to provide a better fit indicating the occurrences of extreme returns in all cryptocurrencies followed by the basic SV model. This model is also found to provide better forecasts than other competing models. Finally, incorporating jump, leverage and moving average components in stochastic volatility do not show significant improvement in forecasting the volatility of cryptocurrencies.

The rest of the paper is organized as follows: Next section discusses the methods employed and the data used in the study. Subsequent section discussed empirical results and the last section concludes the paper.

\section{MATERIALS AND METHODS}

\section{STOCHASTIC VOLATILITY MODELS}

The first model we consider is the basic stochastic volatility (SV) model 


$$
\begin{aligned}
& r_{t}=\mu+\varepsilon_{t}^{r}, \quad \varepsilon_{t}^{r} \sim N\left(0, e^{h_{t}}\right), \\
& h_{t}=\mu_{h}+\phi_{h}\left(h_{t-1}-\mu_{h}\right)+\varepsilon_{t^{h}}, \quad \varepsilon_{t}^{h} \sim N\left(0, \omega_{h}^{2}\right),
\end{aligned}
$$

where $r_{t}$ is the log-return at time $t, h_{t}$ is the conditional log-volatility assumed to follow a stationary autoregressive $\operatorname{AR}(1)$ process with persistent parameter $|\phi|<1$. The process is initialized with $h_{1} \sim N\left(\mu_{h}, \omega_{h}^{2} /\left(1-\phi_{h}^{2}\right)\right)$, where $\mu_{h}$ is the unconditional mean and $\varepsilon_{t}^{h}, \varepsilon_{t}^{r}$ are independent of each other.

The second model assumes heavy-tailed innovations for returns. Here we focus on the model with Student- $t$ innovations. This model $(\mathrm{SV}-t)$ is defined as,

$$
r_{t}=\mu+\varepsilon_{t}^{r}, \varepsilon_{t}^{r} \sim t_{v}\left(0, e^{h_{t}}\right)
$$

whereas in the basic SV model, the similar stationary AR(1) process as in (2) was assumed for conditional $\log$-volatility $h_{t}$. Since the tails of Student- $t$ are heavier than the Gaussian distribution, more extreme observations are allowed in this setup compared to the basic SV model.

Next, we consider the SV model with jumps (SV-J). Large changes in the cryptocurrencies prices may be modeled by incorporating the probability of rare jumps in the model. The SV-J model is defined as

$$
r_{t}=\mu+k_{t} q_{t}+\varepsilon_{t}^{r}, \quad \varepsilon_{t}^{r} \sim N\left(0, e^{h_{t}}\right)
$$

where again the same stationary AR(1) process as in (2) is considered for the conditional log-volatility $h_{t}, q_{t}$ is a binary jump variable with success probability $\mathrm{P}\left(q_{t}=1\right)=\kappa$. $q_{t}=1$, at time $t$ when a jump occurs and the size of the jump is determined by $k_{t}$, where $k_{t} \sim N\left(\mu_{k}, \sigma_{k}^{2}\right) \cdot \mu_{k}$ is the average size of the jump.

The fourth stochastic volatility model that allows for a leverage effect is the asymmetric stochastic volatility model or SV-L model and defined as

$$
\begin{aligned}
& r_{t}=\mu+\varepsilon_{t}^{r}, \varepsilon_{t}^{r} \sim N\left(0, e^{h_{t}}\right), \\
& h_{t}=\mu_{h}+\phi_{h}\left(h_{t-1}-\mu_{h}\right)+\varepsilon_{t}^{h}, \varepsilon_{t}^{h} \sim N\left(0, \omega_{h}^{2}\right),
\end{aligned}
$$

where the innovations $\varepsilon_{t}^{r}$ and $\varepsilon_{t}^{h}$ jointly follows a bivariate normal distribution

$$
\left(\begin{array}{c}
\varepsilon_{t}^{r} \\
\varepsilon_{t}^{h}
\end{array}\right) \sim N\left(0,\left(\begin{array}{cc}
e^{h_{t}} & \rho e^{\frac{1}{2} h_{t}} \omega_{h} \\
\rho e^{\frac{1}{2} h_{t}} \omega_{h} & \omega_{h}^{2}
\end{array}\right)\right)
$$

With $\rho=10$ the SV-L model is the basic SV model. If a negative shock at $r_{t-1}$ occurred $(\rho<0)$, the log-volatility at time $t$ is likely to be larger.

The next model under consideration incorporates conditional volatility in the mean equation. This SV-M model is defined as

$$
r_{t}=\mu+\lambda e^{h_{t}}+\varepsilon_{t}^{r}, \varepsilon_{t}^{r} \sim N\left(0, e^{h_{t}}\right)
$$

In this model, the conditional volatility moves in the conditional mean equation as a control variable. This specification allows for the likelihood that the given data series depends on its own conditional volatility. Clearly, this model also reduces to the basic SV model when $\lambda=0$.

The last model is the basic stochastic volatility model with moving averages (SV-MA) innovations. This model is defined as

$$
\begin{aligned}
& r_{t}=\mu+\varepsilon_{t}^{r} \\
& \varepsilon_{t}^{r}=\mu_{t}+\psi \mu_{t-1}, \quad \mu_{t} \sim N\left(0, e^{h_{t}}\right),
\end{aligned}
$$

with $\mu_{0}=0$ and invertibility condition $|\psi|<1$ and the conditional log-volatility $h_{t}$ follows the familiar stationary AR(1) process. This model may be useful for modeling the short-run dynamics of the data as it allows autocorrelation in data over time.

The Bayesian approach was used for the estimation of all SV models. The log-volatilities are jointly sampled as described in Chan (2015). This acceptance-reject Metropolis-Hasting algorithm is based on the precision sampler that instead of the conventional Kalman filter relies on the fast band matrix routines (see Chan \& Jeliazkov 2009 for details of the algorithm).

\section{MODEL SELECTION AND FORECAST EVALUATION}

The Bayes factor was computed as outlined in Chan and Grant (2016). More specifically, let $M_{1}, M_{2}, \ldots, M_{k}$ be the competing models each defined by a likelihood function $p\left(\boldsymbol{r} \mid \boldsymbol{\theta}_{k}, M_{k}\right)$ and a prior density function $p\left(\boldsymbol{\theta}_{k}, M_{k}\right)$, where $\boldsymbol{\theta}_{k}$ is the parameter vector. The Bayes factor is used as a selection criterion for Bayesian model comparison and defined as,

$$
B F_{i j}=p\left(\boldsymbol{r} \mid M_{i}\right) / p\left(\boldsymbol{r} \mid M_{j}\right)
$$

where $p\left(\boldsymbol{r} \mid M_{k}\right)=\int p\left(\boldsymbol{r} \mid \boldsymbol{\theta}_{k}, M_{k}\right) p\left(\boldsymbol{\theta}_{n} \mid M_{k}\right) \mathrm{d} \boldsymbol{\theta}_{k}$ is the marginal likelihood. This marginal likelihood is calculated using an improved version of adaptive importance sampling method of Chan and Eisenstat (2015). This method also known as the cross-entropy method was originally developed by Rubinstein (1997) and further established by Rubinstein and Kroese (2004). Under model $M_{k}, k=i, j$, the observed data is more likely to fit under $M_{i}$ compared to $M_{j}$ if $B F_{i j}>1$ and thus viewed as a piece of evidence in favor of model $M_{i}$.

To evaluate whether two competing sets of forecasts are equally accurate, the Diebold and Mariano (1995) test of equal predictive ability is commonly employed. The predictive ability of the competing models is compared using the popular Diebold-Mariano (DM) test. Let $h_{t}, \hat{h}_{1 t^{\prime}}$ and $\hat{h}_{2 t}$ be the actuals, and the competing forecasts of model 1 and 2 , respectively, and consider a given loss function $L$ (.) that depends on the forecast error $\hat{e}_{i j}, i=1,2$ Let us define the loss differential series $d_{t}=L\left(\hat{e}_{1 t}\right)-L\left(\hat{e}_{2 t}\right)$. 
Typically, the squared loss error $L\left(e_{i}\right)=e_{i,}^{2} i=1,2$ are used. Diebold and Mariano (1995) showed that the null hypothesis of equal predictive accuracy against the alternative that one forecast is superior can be tested using the DM test statistic

$$
D M=\frac{\bar{d}}{\sqrt{\hat{V}(\bar{d})}} \sim N(0,1),
$$

$$
\begin{aligned}
& \text { where }{ }^{\bar{d}=T^{-1} \sum_{t=1}^{T} d_{t}, \text { and }} \sqrt{T}\left(\bar{d}-E\left(d_{t}\right)\right) \stackrel{d}{\rightarrow} N(0, V(\bar{d})), \\
& \hat{V}(\bar{d})=T^{-1}\left(\hat{\gamma}_{0}+2 \sum_{l=1}^{q} w_{l} \hat{\gamma}_{l}\right), q=l-1, w_{l}=1-l /(q+1), \\
& \hat{\gamma}_{i}=T^{-1} \sum_{t=l+1}^{T}\left(d_{t}-\bar{d}\right)\left(d_{t-l}-\bar{d}\right), l=1, \ldots, q
\end{aligned}
$$

and $l$ is the length of the forecast horizon.

To improve the small sample size behavior of the DM test, Harvey et al. (1997) proposed a modified DM (MDM) test,

$$
M D M=\sqrt{T+1-2 l+T^{-1} l(l-1)}\left(\frac{\bar{d}}{\sqrt{\hat{V}(\bar{d})}}\right) .
$$

If the value of MDM statistic is positive $(M D M>0)$, forecasts of Model $_{1}$ would be considered superior to the forecasts of Model $_{2}$, otherwise, Model $_{2}$ is superior to that of Model $_{1}$.

\section{DATA}

The data used in this study consist of daily closing prices (in USD) of four major cryptocurrencies. The data (till
June 01,2018 ) of four of the top ten cryptocurrencies (Bitcoin, Ethereum, Ripple, and Litecoin), ranked by market capitalization in June 2018, were obtained from https:/coinmarketcap.com/. As of June 2018, the market capitalizations of Bitcoin, Ethereum, Ripple, and Litecoin were approximately 140, 50, 26, and 7 billion U.S. dollars, respectively (cryptocoincharts.info). The daily log returns are calculated as $r_{t}=100 \times\left(\log \left(P_{t}\right)-\log \left(P_{t-1}\right)\right), t=1,2, \ldots T$, where $P_{t}$ is the closing price of a cryptocurrency on day $t$.

\section{RESULTS AND DISCUSSION}

Table 1 presents the basic summary statistics of the logreturns of four cryptocurrencies. The mean log-returns of all cryptocurrencies were found close to zero. The standard deviations of Ripple and Ethereum were higher than Litecoin and Bitcoin. All return distributions showed high Kurtosis (fat-tails). These large Kurtosis may be considered as an indication of the presence of extreme returns. Ripple and Litecoin returns showed positive Skewness. The normality in returns was tested using the Jarque-Bera test for normality and high values of this test further confirms the non-normality of returns. These observations indicate fitting alternative distributions that can describe the data better than the normal. The Ljung-Box test for squared returns was found highly significant for all cryptocurrencies, hence indicating dependence in squared returns and the need for fitting volatility models.

Figure 1 shows the daily closing prices and daily logreturns of four major cryptocurrencies. It can be seen from the charts of daily prices that the prices of these cryptocurrencies have increased significantly in 2017. The prices have reached the maximum at the end of 2017 and then dropped afterward. Volatility clustering is clearly visible in the daily log-returns of all cryptocurrencies along with extreme observations. The improved cross-entropy method was used for the calculation of the marginal loglikelihoods (Chan \& Eisenstat 2015; Chan \& Grant 2016).

TABLE 1. Summary statistics of cryptocurrencies log returns

\begin{tabular}{lcccc}
\hline & Bitcoin & Ethereum & Ripple & Litecoin \\
\hline Duration & Apr 28, 2013- & Aug 08, 2015- & Aug 04, 2013- & Apr 28, 2013- \\
& June 01, 2018 & June 01, 2018 & June 01, 2018 & June 018 \\
Sample size & 1860 & 1028 & 1762 & 1860 \\
Minimum & -11.5608 & -13.7000 & -27.7600 & -22.3200 \\
Maximum & 15.5239 & 17.9100 & 44.6200 & 36.0000 \\
Mean & 0.0867 & 0.2801 & 0.1149 & 0.0774 \\
Median & 0.0941 & -0.0176 & -0.1187 & 0 \\
SD & 1.9461 & 3.1057 & 3.4595 & 2.9924 \\
Skewness & -0.1823 & 0.5215 & 2.0056 & 1.7801 \\
Kurtosis & 10.8049 & 7.3898 & 29.8476 & 28.1375 \\
JB & $4731.30^{* * *}$ & $872.01 * * *$ & $54099^{* * *}$ & $49954^{* * *}$ \\
$Q^{2}(20)$ & $660.68^{* * *}$ & $200.80^{* * *}$ & $260.93 * * *$ & $387.36^{* * * *}$ \\
\hline
\end{tabular}

JB: Jarque-Bera test for normality; $Q^{2}(20)$ is the Ljung-Box test on squared returns; ***represents the significance at the $1 \%$ level 
The results of posterior estimates of parameters and marginal log-likelihood along with their standard deviations of all models are reported in Table 2. Similar estimates for the parameters of the conditional variance process were observed. The posterior mean of the persistent parameter $\phi_{h}$ was between 0.90 and 0.99 across the volatility models. Higher values for this parameter is an indication of high persistent stochastic volatility. The SVmodel with $\phi_{h}=0.99$ was found to be the highest persistent model. The degree of freedom parameter $v$ was between 8 and 18, indicating the heavy-tails for the $t$ distribution. This also implies the presence of outliers in all cryptocurrencies. A negative leverage parameter $\rho$ in SV-L model is an indication of asymmetry in returns. The posterior estimates of this parameter were found small indicating that the asymmetric component in the volatility model may not be important for these cryptocurrencies.

The SV-J model is used to measure the jumps in the cryptocurrencies. The average jump size and its standard deviation are denoted by $\mu_{k}$ and $\sigma_{k}$, respectively. The average jump size was found positive and small for Ripple (0.56) and Litecoin (0.51), negative and small for Bitcoin $(-0.39)$ and positive and high for Ethereum (2.72) with small standard deviations. These findings showed that the jump component in the volatility model may be considered important. The parameter $\lambda$ in SV-M model that captures the extent of volatility in the mean equation was found small $(0.01-0.04)$ for all cryptocurrencies. Remember, the SV-M reduces to the basic SV model when $\lambda=0$. Besides, the $95 \%$ credible intervals (not reported here), for this parameter include 0 . Hence, these findings showed that the basic SV model may be preferred over the SV-M model. Similarly, the $\psi$ parameter estimates were also found close to zero, with 0 included in $95 \%$ credible intervals, indicating this parameter may not be important in the SV-MA volatility model.

Next, we report the model comparison results. The log marginal likelihood values are reported in Table 2 .
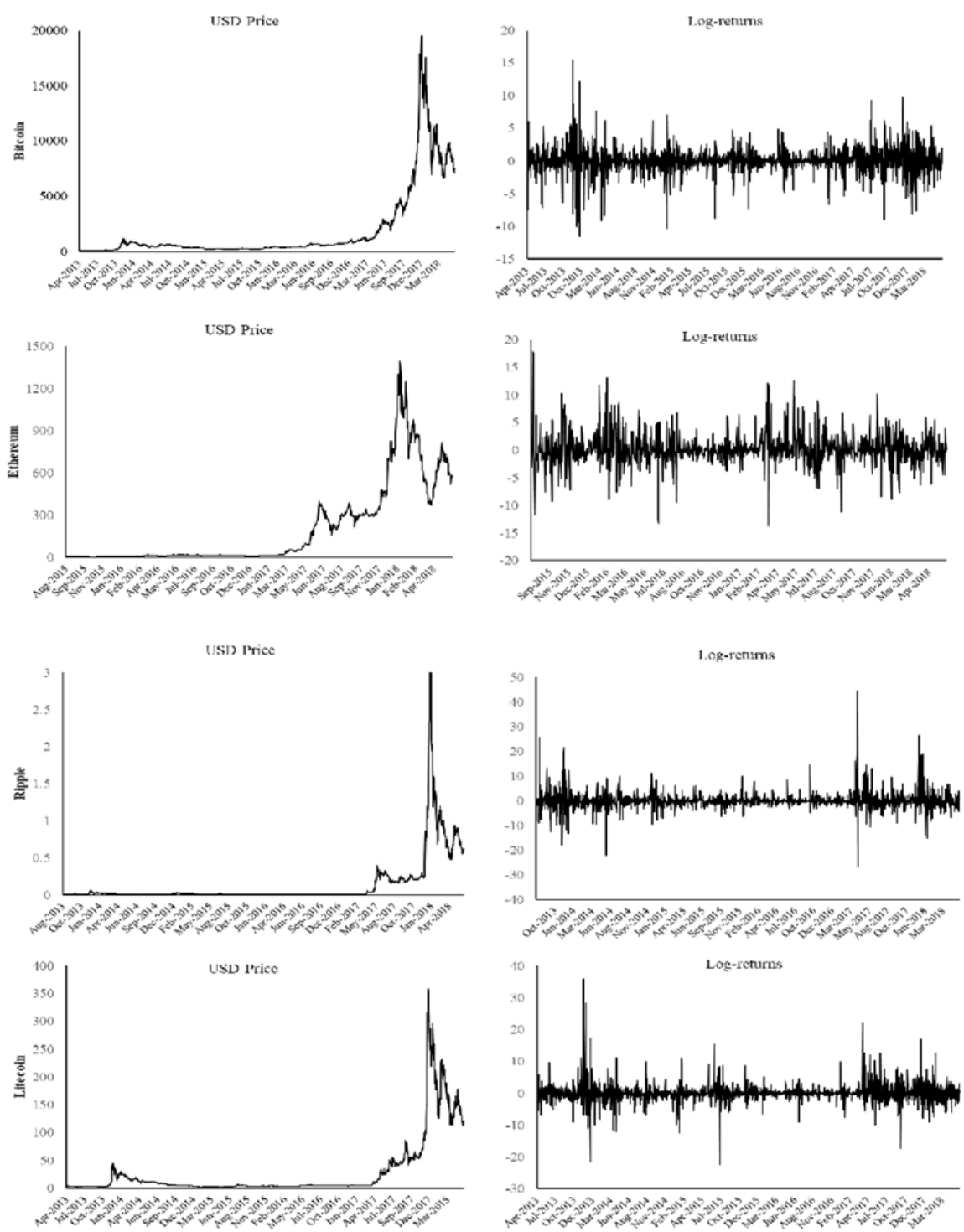

FIGURE 1. Daily prices and log-returns of cryptocurrencies 
TABLE 2. Posterior means and standard deviations (in parenthesis) of parameters of stochastic volatility models

\begin{tabular}{|c|c|c|c|c|c|c|c|c|c|c|c|c|}
\hline & $\mu$ & $\mu_{h}$ & $\phi_{h}$ & $\omega_{h}^{2}$ & $\kappa$ & $\mu_{k}$ & $\sigma_{k}^{2}$ & $\lambda$ & $\psi$ & $v$ & $\rho$ & MLL \\
\hline \multicolumn{13}{|l|}{ Bitcoin } \\
\hline SV & $0.07(0.02)$ & $0.43(0.19)$ & $0.93(0.02)$ & $0.26(0.05)$ & - & - & - & - & - & - & - & $-3332.9(0.26)$ \\
\hline SV- $t$ & $0.06(0.02)$ & $0.30(0.42)$ & $0.97(0.01)$ & $0.11(0.02)$ & - & - & - & - & - & $11.18(2.07)$ & - & $-3303.0(0.15)$ \\
\hline SV-J & $0.08(0.02)$ & $0.27(0.27)$ & $0.96(0.01)$ & $0.16(0.03)$ & $0.09(0.01)$ & $-0.39(0.25)$ & $7.13(1.33)$ & - & - & - & - & $-3355.0(0.73)$ \\
\hline SV- L & $0.06(0.02)$ & $0.40(0.18)$ & $0.93(0.01)$ & $0.28(0.02)$ & - & - & - & - & - & - & $0.01(0.05)$ & $-3335.4(0.28)$ \\
\hline SV-M & $0.06(0.02)$ & $0.43(0.19)$ & $0.94(0.01)$ & $0.25(0.02)$ & - & - & - & $0.01(0.01)$ & - & - & - & $-3339.2(0.21)$ \\
\hline SV-MA & $0.07(0.02)$ & $0.40(0.17)$ & $0.92(0.01)$ & $0.29(0.02)$ & - & - & - & - & $-0.01(0.02)$ & - & - & $-3335.7(0.40)$ \\
\hline \multicolumn{13}{|c|}{ Ethereum } \\
\hline SV & $0.02(0.06)$ & $1.61(0.20)$ & $0.92(0.02)$ & $0.22(0.06)$ & - & - & - & - & - & - & - & $-2429.1(0.12)$ \\
\hline SV- $t$ & $0.01(0.06)$ & $1.51(0.23)$ & $0.93(0.02)$ & $0.18(0.04)$ & - & - & - & - & - & $17.95(2.84)$ & - & $-2427.3(0.05)$ \\
\hline SV-J & $-0.04(0.06)$ & $1.47(0.22)$ & $0.93(0.02)$ & $0.22(0.05)$ & $0.07(0.02)$ & $2.72(0.93)$ & $6.41(3.95)$ & - & - & - & - & $-2428.4(0.26)$ \\
\hline SV- L & $0.01(0.06)$ & $1.60(0.19)$ & $0.90(0.02)$ & $0.27(0.06)$ & - & - & - & - & - & - & $-0.03(0.06)$ & $-2431.0(0.20)$ \\
\hline SV-M & $-0.13(0.08)$ & $1.60(0.18)$ & $0.91(0.02)$ & $0.24(0.05)$ & - & - & - & $0.04(0.01)$ & - & - & - & $-2430.1(0.10)$ \\
\hline SV-MA & $0.02(0.06)$ & $1.61(0.19)$ & $0.91(0.02)$ & $0.26(0.05)$ & - & - & - & - & $0.01(0.03)$ & - & - & $-2432.2(0.12)$ \\
\hline \multicolumn{13}{|l|}{ Ripple } \\
\hline SV & $-0.12(0.03)$ & $1.11(0.24)$ & $0.93(0.03)$ & $0.57(0.03)$ & - & - & - & - & - & - & - & $-3810.9(0.46)$ \\
\hline SV- $t$ & $-0.11(0.04)$ & $1.41(0.50)$ & $0.99(0.02)$ & $0.50(0.14)$ & - & - & - & - & - & $8.05(1.15)$ & - & $-3804.8(0.98)$ \\
\hline SV-J & $-0.10(0.04)$ & $1.10(0.33)$ & $0.95(0.02)$ & $0.26(0.11)$ & $0.03(0.01)$ & $0.56(0.61)$ & $20.20(11.59)$ & - & - & - & - & $-3909.4(0.63)$ \\
\hline SV- L & $-0.11(0.02)$ & $0.96(0.18)$ & $0.90(0.02)$ & $0.55(0.07)$ & - & - & - & - & - & - & $0.04(0.05)$ & $-3812.8(0.32)$ \\
\hline SV-M & $-0.12(0.03)$ & $1.01(0.17)$ & $0.90(0.01)$ & $0.53(0.02)$ & - & - & - & $0.03(0.01)$ & - & - & - & $-3811.0(0.58)$ \\
\hline SV-MA & $-0.11(0.02)$ & $0.95(0.18)$ & $0.89(0.01)$ & $0.62(0.05)$ & - & - & - & - & $0.01(0.02)$ & - & - & $-3811.6(0.56)$ \\
\hline \multicolumn{13}{|l|}{ Litecoin } \\
\hline SV- $t$ & $-0.03(0.02)$ & $0.90(0.18)$ & $0.91(0.01)$ & $0.45(0.07)$ & - & - & - & - & - & - & - & $-3740.5(0.32)$ \\
\hline SV-J & $-0.02(0.03)$ & $1.29(0.51)$ & $0.99(0.01)$ & $0.04(0.01)$ & - & - & - & - & - & $8.56(1.01)$ & - & $-3722.3(0.92)$ \\
\hline SV- L & $-0.03(0.02)$ & $0.81(0.33)$ & $0.96(0.02)$ & $0.19(0.07)$ & $0.06(0.01)$ & $0.51(0.45)$ & $27.78(8.34)$ & - & - & - & - & -3743.8 \\
\hline SV-M & $-0.02(0.02)$ & $0.86(0.16)$ & $0.90(0.01)$ & $0.52(0.03)$ & - & - & - & - & - & - & $0.02(0.02)$ & $-3743.4(0.40)$ \\
\hline SV-MA & $-0.04(0.02)$ & $0.87(0.17)$ & $0.90(0.01)$ & $0.50(0.05)$ & - & - & - & $0.02(0.01)$ & - & - & - & $-3745.9(0.28)$ \\
\hline $\mathrm{SV}-t$ & $-0.03(0.02)$ & $0.89(0.20)$ & $0.90(0.03)$ & $0.48(0.15)$ & - & - & - & - & $-0.09(0.02)$ & - & - & $-3738.2(0.34)$ \\
\hline
\end{tabular}

MLL: Marginal log likelihood 
These values are used to compute the Bayes factor to evaluate the model fit. The results of the Bayes factor are shown in Table 3 where each row denotes the Bayes factors of the model in a row against the models in columns. For example, the first row in Table 3 shows the Bayes factors of SV model against other competing models (SV- $t$, SV-J, SV-L, SV-M, and SV-MA). Bayes factor greater than 1 is the confirmation in support of the model in row compared to the model in column. Overall, the best model is the SV- $t$ model for all four cryptocurrencies considered in this study followed by the basic SV model. These results are in support the findings of parameter estimates where the degree of freedom parameter $v$ was found significant for all cases, indicating the importance of heavy-tailed distribution for returns. Furthermore, these results supported the ranking of the marginal likelihood reported in Table 2. The Bayes factor of $2.6 \times 10^{45}$ in favor of the $\mathrm{SV}-t$ model against the standard SV model indicate strong evidence for the heavy-tailed model. Similar findings were observed when the SV-t model was compared to other volatility models.
We further examine the important features of modeling the dynamics of cryptocurrencies. The basic SV model was found to provide better fit than other Gaussian volatility models except for Ethereum where SV-J and Litecoin where the SV-MA models slightly showed a better fit. For example, the Bayes factor $5.91 \times 10^{9}$ (Bitcoin) was in favor of the basic SV model against the SV-L model indicating that the returns may not show any leverage or asymmetry. The Bayes factor of the SV-J model against each SV-L, SV-M and SV-MA model was greater than 1 (Ethereum). This supports our estimation results of Table 2 where the average jump size for Ethereum was found the largest. Overall, the results of model fit showed that incorporating heavy-tail distribution has improved the estimation and that jumps and asymmetric components may not be important for the volatility dynamics of these cryptocurrencies.

The out of sample forecast of volatility is of vital importance for portfolio and risk management and also for investors who desire to measure the performance of the model. The data set was divided into two parts: the in-

TABLE 3. Model fit evaluation under Bayesian model comparison

\begin{tabular}{|c|c|c|c|c|c|}
\hline & \multicolumn{5}{|c|}{ Bitcoin } \\
\hline & $\mathrm{SV}-t$ & SV-J & SV- L & SV-M & SV-MA \\
\hline SV & $1.03 \mathrm{E}-13$ & $3.96 \mathrm{E}+09$ & $5.91 \mathrm{E}+09$ & $5.45 \mathrm{E}+02$ & $1.64 \mathrm{E}+01$ \\
\hline $\mathrm{SV}-t$ & 1 & $2.67 \mathrm{E}+45$ & $1.18 \mathrm{E}+14$ & $5.27 \mathrm{E}+15$ & $1.59 \mathrm{E}+14$ \\
\hline SV-J & & 1 & $3.07 \mathrm{E}-09$ & $1.37 \mathrm{E}-07$ & $4.15 \mathrm{E}-09$ \\
\hline SV- L & & & 1 & $4.47 \mathrm{E}+01$ & $1.34 \mathrm{E}+00$ \\
\hline \multirow[t]{3}{*}{ SV-M } & & & & 1 & $3.02 \mathrm{E}-02$ \\
\hline & \multicolumn{5}{|c|}{ Ethereum } \\
\hline & $\mathrm{SV}-t$ & SV-J & SV-L & SV-M & SV-MA \\
\hline SV & $1.65 \mathrm{E}-01$ & $4.97 \mathrm{E}-01$ & $6.69 \mathrm{E}+00$ & $2.72 \mathrm{E}+00$ & $2.22 \mathrm{E}+01$ \\
\hline $\mathrm{SV}-t$ & 1 & $3.00 \mathrm{E}+00$ & $4.04 \mathrm{E}+01$ & $1.64 \mathrm{E}+01$ & $1.34 \mathrm{E}+02$ \\
\hline SV-J & & 1 & $1.35 \mathrm{E}+01$ & $5.47 \mathrm{E}+00$ & $4.47 \mathrm{E}+01$ \\
\hline SV- L & & & 1 & $4.07 \mathrm{E}-01$ & $3.32 \mathrm{E}+00$ \\
\hline \multirow[t]{3}{*}{ SV-M } & & & & 1 & $8.17 \mathrm{E}+00$ \\
\hline & \multicolumn{5}{|c|}{ Ripple } \\
\hline & $\mathrm{SV}-t$ & SV-J & SV- L & SV-M & SV-MA \\
\hline SV & $2.20 \mathrm{E}-03$ & $5.99 \mathrm{E}+42$ & $2.01 \mathrm{E}+00$ & $1.11 \mathrm{E}+00$ & $2.01 \mathrm{E}+00$ \\
\hline $\mathrm{SV}-t$ & 1 & $2.67 \mathrm{E}+45$ & $2.98 \mathrm{E}+03$ & $4.93 \mathrm{E}+02$ & $8.97 \mathrm{E}+02$ \\
\hline SV-J & & 1 & $1.11 \mathrm{E}-42$ & $1.842 \mathrm{E}-43$ & $3.36 \mathrm{E}-43$ \\
\hline SV- L & & & 1 & $1.65 \mathrm{E}-01$ & $3.01 \mathrm{E}-01$ \\
\hline \multirow[t]{3}{*}{ SV-M } & & & & 1 & $1.82 \mathrm{E}+00$ \\
\hline & \multicolumn{5}{|c|}{ Litecoin } \\
\hline & $\mathrm{SV}-t$ & SV-J & SV- L & SV-M & SV-MA \\
\hline SV & $1.25 \mathrm{E}-08$ & $2.71 \mathrm{E}+01$ & $18.17 \mathrm{E}+00$ & $2.21 \mathrm{E}+02$ & $10.03 \mathrm{E}-01$ \\
\hline $\mathrm{SV}-t$ & 1 & $2.17 \mathrm{E}+09$ & $1.46 \mathrm{E}+09$ & $1.78 \mathrm{E}+10$ & $8.04 \mathrm{E}+06$ \\
\hline SV-J & & 1 & $6.70 \mathrm{E}-01$ & $8.16 \mathrm{E}+00$ & $3.70 \mathrm{E}-03$ \\
\hline SV- L & & & 1 & $12.18 \mathrm{E}+00$ & $5.52 \mathrm{E}-03$ \\
\hline SV-M & & & & 1 & $4.53 \mathrm{E}-04$ \\
\hline
\end{tabular}

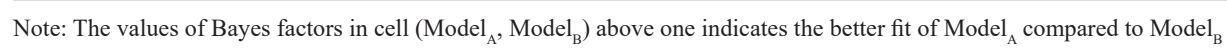


sample period of initial $N$ observations were used for the estimation of parameters and remaining $K=T-N$ observations for volatility forecasts. The last one-year data (July 1, 2017 - June 1, 2018), approximately 336 observations, were left for forecast evaluation. One step ahead forecasts are generated from stochastic volatility models using a rolling window scheme. More specifically, initial $N$ observations are used for the estimation of the model and one step ahead forecast of volatility was generated, then the sample is rolled forward one day, discarding the first observation, the model is re-estimated and one step ahead forecast is generated. In this way, $K$ one step ahead forecasts of volatility are generated and compared with the daily squared returns (proxy for true volatility). Similarly, the weekly and monthly forecasts of volatility were also generated.

Table 4 presents the MDM test results for one step ahead volatility forecasts of the six stochastic volatility models. The value of MDM $>0$ indicates the forecasting accuracy and superiority of the model in column against the model in row. For example, Cell $(1,2)$ of Table 4 represents the value of MDM test statistic (7.87) for model SV- $t$ against SV. This higher positive number shows that the SV- $t$ model has better forecasts of volatility than the standard SV model. The best forecast model for each cryptocurrency is denoted by $(*)$. The results of Table 4 show the superior forecast performance of SV- model than all other competing volatility models. The basic SV model may be considered the second-best based on the values of MDM statistic. This shows that incorporating heavy-tails in volatility model not only improves the estimates of the parameters but produce accurate forecasts of volatility. These findings support the results of Table 3 where SV- $t$ model was found the best-fitted model for these cryptocurrencies followed by the basic SV models. The results of one week and one month ahead forecasts (not reported here for brevity) largely affirm the superior performance of heavy-tailed stochastic volatility model.

Some broad conclusions can be drawn from this research: The SV models may be considered as a better

TABLE 4. MDM test for one day ahead volatility forecast

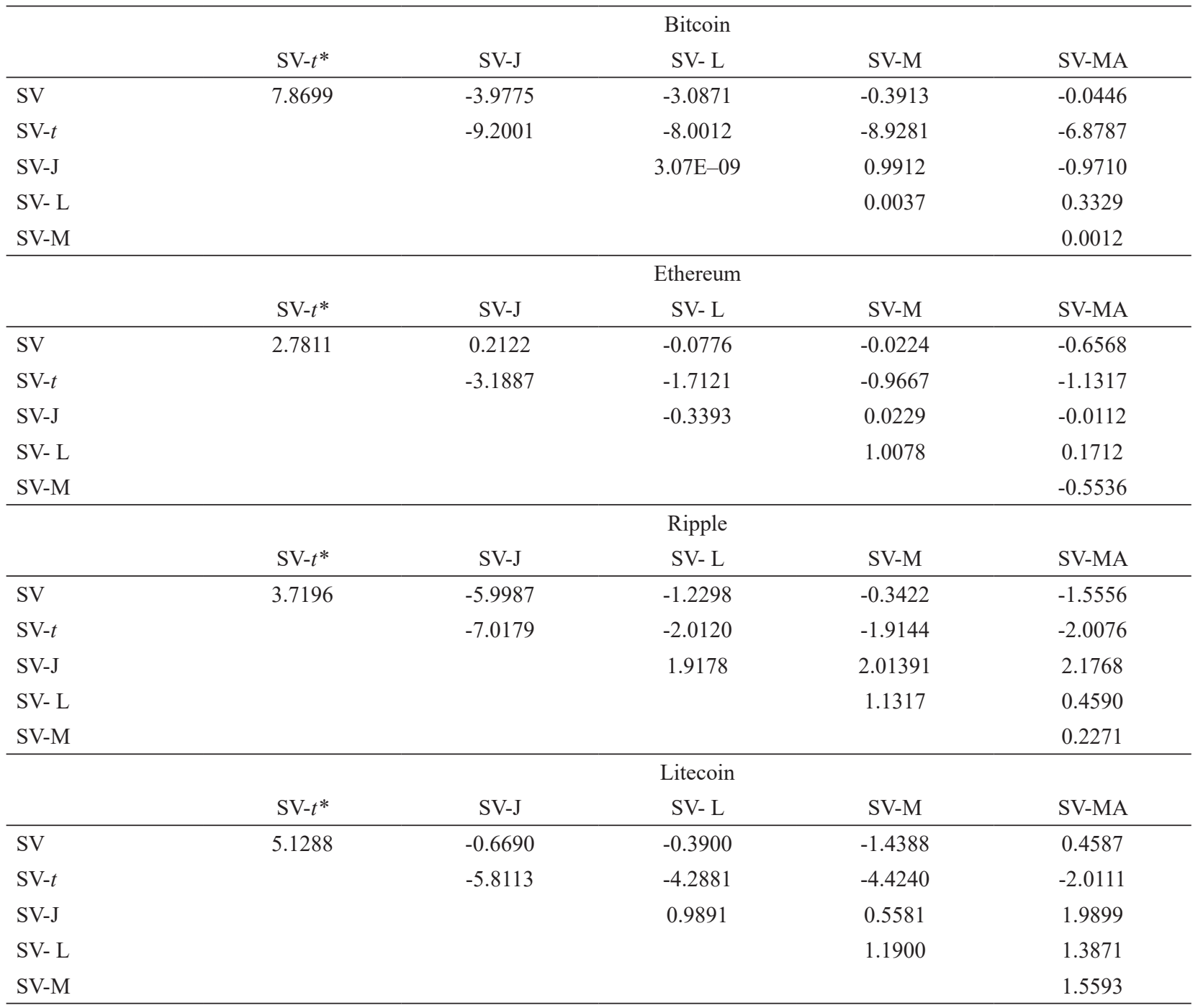

Note: Value of cell $\left(\mathrm{Model}_{\mathrm{A}}, \mathrm{Model}_{\mathrm{B}}\right)$ above zero indicates that $\operatorname{Model}_{\mathrm{A}}$ has Better fit than $\operatorname{Model}_{\mathrm{B}}$. '*` represents the model with best predictive accuracy 
alternative to the traditional GARCH-type models. The uncertainty in the parameters can be modeled using the Bayesian approach. The four major cryptocurrencies show almost the same volatility dynamics and that the heavytailed stochastic volatility models provided better insample and out-of-sample results. The leverage effect, an important feature in stock prices, was not found important in cryptocurrencies.

\section{CONCLUSION}

This study models and forecasts the volatility of four major cryptocurrencies (Bitcoin, Ethereum, Ripple, and Litecoin) using a number of stochastic volatility models. The models were estimated using the Bayesian approach and the model fit was evaluated using the marginal likelihood and Bayes factor. The one-step-ahead forecasts of volatility were evaluated using the modified Diebold-Mariano test. The heavy-tailed stochastic volatility (SV-t) model was found to better fit the data indicating the occurrences of extreme returns in all cryptocurrencies followed by the basic SV model. The forecasting performance of this model was also found superior to the competing models. The jump, leverage and moving average components were not found significant for most cryptocurrencies studied in this research. The findings of this study may be considered important for risk and portfolio management where reliable estimates are desired. However, this study modeled and forecasted the volatility of only four major cryptocurrencies. The research may be extended to include other popular cryptocurrencies for better understanding the volatility dynamics in the crypto-market.

\section{REFERENCES}

Baur, D.G. \& Dimpfl, T. 2018. Asymmetric volatility in cryptocurrencies. Economic Letters 173: 148-151.

Bezerra, P.C.S. \& Albuquerque, P.H.M. 2017. Volatility forecasting via SVR-GARCH with mixture of Gaussian kernels. Computational Management Science 14(2): 179196.

Bollerslev, T. 1986. Generalized autoregressive conditional heteroskedasticity. Journal of Econometrics 31(3): 307-327.

Borri, N. 2019. Conditional tail-risk in cryptocurrency markets. Journal of Empirical Finance 50: 1-19.

Bouri, E., Azzi, G. \& Dyhrberg, A.H. 2017. On the returnvolatility relationship in the Bitcoin market around the price crash of 2013. Economics: The Open-Access, OpenAssessment E-Journal 11: 1-16.

Caporale, G.M. \& Zekokh, T. 2019. Modelling volatility of cryptocurrencies using markov-switching GARCH models. Research in International Business and Finance 48: 143155.

Catania, L. \& Grassi, S. 2017. Modelling crypto-currencies financial time-series. CEIS Working Paper No. 417. Available at SSRN: https://ssrn.com/abstract=3084109.

Catania, L., Grassi, S. \& Ravazzolo, F. 2018. Predicting the volatility of cryptocurrency time-series. CAMP Working Paper Series 3.

Chan, J.C.C. 2015. The stochastic volatility in mean model with time-varying parameters: An application to inflation modelling. Journal of Business and Economic Statistics 35(1): $17-28$

Chan, J.C.C. 2013. Moving average stochastic volatility models with applications to inflation forecast. Journal of Econometrics 176(2): 162-172.

Chan, J.C.C. \& Grant, A.L. 2016. Modeling energy price dynamics: GARCH versus stochastic volatility. Energy Economics 54: 182-189.

Chan, J.C.C. \& Eisenstat, E. 2015. Marginal likelihood estimation with cross-entropy method. Economics Review 34(3): 256-285.

Chan, J.C.C. \& Jeliazkov, I. 2009. Efficient simulation and integrated likelihood estimation in state space models. International Journal of Mathematical Modelling and Numerical Optimisation 1: 101-120.

Charfeddine, L. \& Maouchi, Y. 2018. Are shocks on the returns and volatility of cryptocurrencies really persistent? Finance Research Letters 28: 423-430.

Charle, A. \& Darne-Lemna, O. 2018. Volatility estimation for bitcoin: Replication and robustness. International Economics 157: 23-32.

Cheong, C.W., Lai, N.S., Isa, Z. \& Nor, A.H.S.M. 2012. Asymmetry dynamic volatility forecast evaluations using interday and intraday data. Sains Malaysiana 14(10): 12871299.

Chu, J., Chan, S., Nadarajah, S. \& Osterrieder, J. 2017. GARCH modelling of cryptocurrencies. Journal of Risk and Financial Management 10(4): 17. doi:10.3390/jrfm10040017.

Creal, D., Koopman, S.J. \& Lucas, A. 2013. Generalized autoregressive score models with applications. Journal of Applied Econometrics 28(5): 777-795.

Diebold, F.X. \& Mariano, R.S. 1995. Comparing predictive accuracy. Journal of Business and Economic Statistics 13(3): 253-263.

Eraker, B., Johannes, M. \& Polson, N. 2003. The impact of jumps in volatility and returns. Journal of Finance 58(3): 1269-1300.

Fakhfekh, M. \& Jeribi, A. 2020. Volatility dynamics of cryptocurrencies' returns: Evidence from asymmetric and long memory GARCH models. Research in International Business and Finance 51: 101075.

Ghysels, E., Harvey, A.C. \& Renault, E. 1996. Stochastic volatility. In Statistical Models in Finance, edited by Rao, C.R. \& Maddala, G.S. North-Holland, Amsterdam; Elsevier. pp. 119-191.

Gkillas, K. \& Katsiampa, P. 2018. An application of extreme value theory to cryptocurrencies. Economics Letters 164: 109-111.

Harvey, A.C. 2013. Dynamic Models for Volatility and Heavy Tails: With Applications to Financial and Economic Time Series. Volume 52. New York: Cambridge University Press.

Harvey, A.C. \& Shephard, N. 1996. The estimation of an asymmetric stochastic volatility model for asset returns. Journal of Business and Economic Statistics 14: 429-434.

Harvey, D., Leybourne, S. \& Newbold, P. 1997. Testing the equality of prediction mean squared error. International Journal of Forecasting 13(2): 281-291.

Katsiampa, P. 2017. Volatility estimation for bitcoin: A comparison of GARCH models. Economics Letters 158: 3-6.

Koopman, S.J. \& Hol Uspensky, E. 2002. The stochastic volatility in mean model: Empirical evidence from international stock markets. Journal of Applied Econometrics 17(6): 667-689. 
Liesenfeld, R. \& Jung, R.C. 2000. Stochastic volatility models: Conditional normality versus heavy-tailed distributions. Journal of Applied Econometrics 15: 137-160.

Liu, R., Shao, Z., Wei, G. \& Wang, W. 2017. GARCH model with fat-tailed distributions and bitcoin exchange rate returns. Journal of Accounting, Business and Finance Research 1(1): 71-75.

Naimy, V.Y. \& Hayek, M.R. 2018. Modelling and predicting the bitcoin volatility using GARCH models. International Journal of Mathematical Modelling and Numerical Optimisation 8(3): 197-215.

Peng, Y., Albuquerque, P.H.M., de Sa, J.M.C., Padula, A.J.A \& Montenegro, M.R. 2018. The best of two worlds: Forecasting high frequency volatility for cryptocurrencies and traditional currencies with support vector regression. Expert Systems with Applications 97: 177-192.

Rahim, M.A.A., Zahari, S.M. \& Shariff, S.R. 2018. Variance targeting estimator for GJR-GARCH under Model's Misspecification. Sains Malaysiana 47(9): 2195-2204.

Shephard, N. 1996. Statistical aspects of ARCH and stochastic volatility. In Time Series Models in Econometrics, Finance and Other Fields, edited by Cox, D.R., Hinkley, D.V. \& Barndorff-Nielson, O.E. London: Chapman \& Hall. pp. 1-67.

Stavroyiannis, S. \& Babalos, V. 2017. Dynamic Properties of the Bitcoin and the US market. Accessed May 10, 2018. https://ssrn.com/abstract=2966998.

Taylor, S.J. 1986. Modelling Financial Time Series. Chichester: John Wiley.

Urquhart, A. 2017. The volatility of Bitcoin. SSRN Electronic Journal. DOI: 10.2139/ssrn.2921082.

Department of Statistics

University of Balochistan

Quetta

Pakistan

*Corresponding author; email: farhatiqb@gmail.com

Received: 8 April 2019

Accepted: 10 November 2019 